\title{
The emerging problem of bacterial resistance in cancer patients; proceedings of a workshop held by MASCC "Neutropenia, Infection and Myelosuppression" Study Group during the MASCC annual meeting held in Berlin on 27-29 June 2013
}

\author{
Bernardo Rapoport $^{1}$ • Jean Klastersky ${ }^{2} \cdot$ Harry Raftopoulos $^{3} \cdot$ Allison Freifeld $^{4}$. \\ Mickael Aoun $^{2} \cdot$ Stephen H. Zinner ${ }^{5,6} \cdot$ Kenneth V.I. Rolston $^{7}$
}

Received: 18 June 2015 /Accepted: 21 March 2016/Published online: 20 April 2016

(C) Springer-Verlag Berlin Heidelberg 2016

\section{Introduction}

Over the past several decades, the successful treatment of fever and presumed infections in neutropenic patients with cancer has been based on the selection of a number of empiric regimens that contain one or more antibiotics. However, today, the successful treatment of these patients is becoming ever more challenging due to the emergence and dissemination of antibiotic-resistant Gram-positive and Gram-negative bacteria. This review summarizes and updates the proceedings of a workshop organized by the MASCC "Neutropenia, Infection and Myelosuppression" Study Group that was presented at the MASCC annual meeting in Berlin 27-29 June 2013.

Bernardo Rapoport

brapoport@rosebankoncology.co.za

Medical Oncology Centre of Rosebank, Johannesburg, South Africa

2 Institut Jules Bordet, Centre des Tumeurs de l'Université Libre de Bruxelles, Brussels, Belgium

Merck and Co, Kenilworth, NJ 07033, USA

4 Nebraska Medical Center, Omaha, NE, USA

5 Department of Medicine, Mount Auburn Hospital, Cambridge, MA, USA

6 Harvard Medical School, Boston, MA, USA

7 Infectious Disease Department, MD Anderson Cancer Center, Houston, TX, USA
Bacterial infections in cancer patients: spectrum of bacterial pathogens

Over the past several decades, substantial shifts in the spectrum of bacterial bloodstream isolates have occurred. Gram-positive cocci, including coagulase-negative staphylococci (CoNS) primarily, and also Staphylococcus aureus, viridans group streptococci, and enterococci predominated in the 1980s and 1990s. Gram-negative bacteria including Enterobacteriaceae (Escherichia coli, Klebsiella sp., Enterobacter sp.) and the non-fermenters (Pseudomonas aeruginosa, Acinetobacter spp., Stenotrophomonas maltophilia) were less commonly isolated. Two recent systematic reviews of bacteremia in febrile neutropenic (FN) patients at several global sites have documented three emerging trends in these patients: (1) Gramnegative isolates are becoming nearly as prevalent (or more so in some areas) as Gram-positives, (2) Gram-positive and Gram-negative bacteria that are resistant to empirically recommended $\beta$-lactam antibiotics are increasing worldwide, and (3) substantial geographic/regional differences exist in patterns of bloodstream isolates from $\mathrm{FN}$ patients $[1,2]$.

\section{Emergence of antibiotic-resistant pathogens}

The majority of drug-resistant infections in hospitalized patients have been grouped under the acronym ESKAPE, which stands for Enterococcus faecium, S. aureus, Klebsiella pneumoniae, Acinetobacter baumannii, P. aeruginosa, and Enterobacter spp. [3]. To adapt this framework to the neutropenic patient, E. coli and S. maltophilia also should be included, yielding the "ESKAPEES" acronym [4]. Of these six Gram-negative and two Gram-positive species with a 
propensity for antibiotic resistance, rates of resistance vary widely among regional and even local institutions. For a given FN patient at risk for bacteremia, the background level of antibiotic resistance in the local environment is the most important risk factor for acquisition of resistant pathogens. Thus, physicians must familiarize themselves with local antibiograms for their particular hospital(s) and choose drugs accordingly. Notably, among 1148 episodes of bacteremia in cancer patients from a prospective multicenter study in Spain, 392 (34 \%) were caused by ESKAPE pathogens (E. coli and Stenotrophomonas excluded in this study), and 54 episodes (4.7 \%) were due to antibiotic-resistant ESKAPE strains [5]. In most studies, E. coli and Klebsiella spp. remain the most frequent causes of bacteremia in FN patients in the current era and an increasing proportion carry resistance plasmids coding for enzymes that destroy the cornerstone antibiotics for empiric therapy of $\mathrm{FN}$ - cephalosporins and carbapenems.

\section{Mechanisms of resistance: Gram-positive organisms}

The Gram-positive pathogens most commonly isolated from neutropenic patients include coagulase-negative staphylococci (CoNS), S. aureus, Enterococcus species, and the viridans group streptococci (VGS) or alpha-hemolytic streptococci [6]. Due to the production of beta-lactamases, virtually all strains of CoNS and S. aureus are resistant to natural penicillins, aminopenicillins, and anti-pseudomonal penicillins The $m e c \mathrm{~A}$ gene encoding low-affinity penicillin-binding protein PBP2a confers resistance to methicillin and other betalactams. Currently $>90 \%$ of CoNS isolates and 40 to $80 \%$ of $S$. aureus isolates are methicillin-resistant. Resistance to other antimicrobial classes (fluoroquinolones, macrolides, tetracyclines, rifampin, and aminoglycosides) also occurs. Resistance to linezolid is conferred primarily by the $c f r$ gene [7]. The mechanisms of daptomycin resistance in S. aureus are varied and include accumulation of single-nucleotide polymorphisms in the multipeptide resistance factor gene ( $\mathrm{mpr}$ F) and cell wall thickening [8]. The mechanism of high-level resistance to vancomycin among $S$. aureus isolates (VISAMIC $\geq 64.0 \mu \mathrm{g} / \mathrm{mL}$ ) involves the horizontal transfer of a transposon containing van $\mathrm{A}$ and associated genes from vancomycin-resistant enterococci (VRE). Vancomycin resistance among Enterococcus species (primarily E. faecium) is mediated by two classes of related gene clusters ( $\operatorname{van} \mathrm{A}$ and van $\mathrm{B}$ ), which produce resistance by altering the target for vancomycin from $\mathrm{D}$-alanine-D-alanine to $\mathrm{D}$-alanine-Dlactate [9]. Penicillin resistance is not uncommon in VGS and Streptococcus pneumoniae, but has not been demonstrated in group A beta-hemolytic streptococci [10]. Tolerance (MBC $\geq 32$ times the MIC) to vancomycin and other agents is also an emerging problem [11].

With the emergence of methicillin-resistant $S$. aureus (MRSA) and penicillin-resistant streptococci, vancomycin has become the agent of choice for the treatment of these and other resistant Gram-positive pathogens in neutropenic patients. Recent changes including the so-called upward "MIC creep" and the development of heteroresistant organisms have undermined the therapeutic potential of this agent. Fortunately, several newer agents with potent Gram-positive activity including linezolid, tedizolid, daptomycin, telavancin, dalbavancin, oritavancin, and ceftaroline have been introduced, and several more are in various stages of development.

\section{Mechanisms of resistance: Gram-negative organisms}

The Gram-negative pathogens most frequently isolated from neutropenic patients include E. coli, Klebsiella species, other Enterobacteriaceae, P. aeruginosa, and other nonfermentative Gram-negative bacilli (NFGNB) $[12,13]$. Antimicrobial agents commonly used for empiric and/or targeted therapy of these infections include the extendedspectrum cephalosporins (e.g., cefepime) and the carbapenems (imipenem, meropenem, and, to a lesser extent, doripenem) and combination agents such as piperacillin/tazobactam. The aminoglycosides are used much less often. The fluoroquinolones are still widely used for chemoprophylaxis in neutropenic patients. Resistance to aminoglycosides results from the production of various aminoglycoside-modifying enzymes that produce acetylation, adenylation, and phosphorylation. Quinolone resistance among E. coli, P. aeruginosa, and other Gram-negative bacilli has been reported from multiple institutions that use these agents for prophylaxis [14]. Mutations in the gyr A and $\operatorname{par} \mathrm{C}$ genes are the most common mechanisms involved in high-level quinolone resistance.

Resistance to the $\beta$-lactams is mediated primarily through the production of a variety of $\beta$-lactamases including Ambler class $\mathrm{C}(\mathrm{AmpC})$ beta-lactamases, and the extended-spectrum $\beta$-lactamases (ESBLs). ESBLs are derived from older, plasmid-mediated hydrolyzing enzymes, primarily the TEM and SHV types, through genetic mutations that broaden their activity spectrum. Cocarriage of other antibiotic resistance coding genes (i.e., to fluoroquinolones, amioglycosides, macrolides, carbapenems, etc.) on the same plasmid can confer a multidrug resistance phenotype in a subset of these pathogens [15].

The carbapenems, previously considered the last line of defense against organisms resistant to other $\beta$-lactams, are currently under threat because of the development and rapid spread of carbapenemases, which belong to Ambler classes A, $\mathrm{B}$, and D [16]. Examples of these carbapenemases include the Klebsiella pneumoniae Carbapenemase (KPC) and the metallo-beta-lactamases New-Delhi-1 and New-Delhi-2, VIM, IMP, and OXA-48. Multiple resistance mechanisms may be present in the same isolate. A detailed discussion of the various resistance mechanisms is outside the scope of this manuscript (common mechanisms of resistance have been 
summarized in Table 1). Nevertheless, the rapid spread of these organisms globally, aided by the convenience of air travel, and the increasing rates of medical tourism, is of great concern. The conduct of periodic surveys to detect epidemiologic changes and the emergence of newer mechanisms of resistance cannot be stressed enough, particularly since institutional and geographic variations are relatively common.

A new gene mediating resistance to colistin ( $m c r-1)$ has been described recently on a multidrug-resistant plasmid [17]. This development could prove very dangerous to neutropenic patients infected with multidrug-resistant Gram-negative bacteria.

\section{Epidemiology of resistant Gram-positive bacterial infections}

Methicillin-resistant S. aureus (MRSA) has been increasing in prevalence over the past two decades. In the USA, community-acquired MRSA is responsible for $80 \%$ or more of skin and soft tissue infections. Most of these infections are caused by a single clone, the most common of which is Strain Type USA 300 (ST USA300) [18]. These strains are currently causing hospital-acquired as well as community-acquired infections in America. This organism is less common in Europe, but a systematic review of data from the mid to late $2000 \mathrm{~s}$ among 14 hematology/oncology centers in eight countries shows over half of all bacteremic $S$. aureus isolates were MRSA, but the range was 18 to $100 \%$ in adults and 0 to $26 \%$ in children [2]. The risk of MRSA infection in neutropenic patients should be assessed according to the clinical presentation and local epidemiology [19]. Regarding outcomes, a single-center study at MD Anderson in Texas, of 223 cases of MRSA bacteremia in cancer patients, reported a $12 \%$ mortality rate and a $52 \%$ vancomycin treatment failure rate. There was a significant association between mortality rates and vancomycin MIC $\geq 2 \mu \mathrm{g} / \mathrm{mL}$ [20].

Vancomycin-resistant enterococci (VRE) are an important cause of bacteremia in neutropenic patients with allohematopoietic stem cell transplantation (HSCT) and hematologic malignancy (HM) [21]. The majority are caused by $E$. faecium and less frequently by Enterococcus faecalis or other enterococci. Prior colonization (OR 3.88; 95 \% CI 1.5-10.4; $p=0.005)$ and T cell depletion (OR 10.89; $95 \%$ CI 1.30 $91.35 ; p=0.028$ ) are important risk factors. Attributable mortality varies between 9 and $14 \%$.

Antibiotic resistance among viridans group streptococci (VGS) varies between institutions and countries. Recent studies in neutropenic patients with HM or HSCT reported 4 to $14 \%$ of VGS isolates being highly resistant to penicillin [22, 23]. Streptococcus mitis is most consistently resistant to penicillin. It is more frequent in children, where 50 to $86 \%$ of strains were found to be penicillin-resistant [24].

Table 1 Common resistance mechanisms among Gram-positive and Gram-negative bacteria causing infections in cancer patients

\begin{tabular}{|c|c|c|}
\hline Organisms & Antimicrobial agents & Resistance mechanisms \\
\hline \multirow{5}{*}{$\begin{array}{l}\text { Staphylococcus species-including } \\
\text { CoNS and S. aureus }\end{array}$} & Penicillin & Beta-lactamase production \\
\hline & Methicillin & Altered PBP2 binding site \\
\hline & Vancomycin & $\begin{array}{l}\text { Multiple mutations, thickened cell wall, vanA- or vanB- } \\
\text { mediated resistance }\end{array}$ \\
\hline & Daptomycin & $\begin{array}{l}\text { Cell membrane mutations, cell wall thickening, reduced } \\
\text { surface binding, polymorphism in the } m p r F \text { gene }\end{array}$ \\
\hline & Linezolid & Mutations at the 23S rRNA binding site, efflux pump ( $c f r$ ) \\
\hline \multirow[t]{4}{*}{ Enterococcus species } & Penicillin/ampicillin & Altered transpeptidase binding sites, overexpression of PBP5 \\
\hline & Gentamicin & Aminoglycoside modifying enzymes, diminished drug entry \\
\hline & Vancomycin & $\begin{array}{l}\text { vanA- or } \operatorname{van} B \text {-mediated resistance (alteration from D-alanine- } \\
\text { D-alanine to D-alanine-D-lactate) }\end{array}$ \\
\hline & Linezolid & 23S rRNA mutations, $c f r$ plasmid-mediated resistance \\
\hline \multirow{4}{*}{$\begin{array}{l}\text { Enterobacteriaceae -including E. } \\
\text { coli, Klebsiella species, } \\
\text { Enterobacter species, and other } \\
\text { Enterobacteriaceae } \\
\text { Non-fermentative Gram-negative } \\
\text { bacilli (NFGNB) including } \\
\text { Acinetobacter } \text { species, } \\
\text { Pseudomonas aeruginosa, } \\
\text { Stenotrophomonas maltophilia }\end{array}$} & $\begin{array}{l}\text { Beta-lactams including penicillins, } \\
\text { cephalosporins, and carbapenems }\end{array}$ & $\begin{array}{l}\text { Altered PBPs, augmented drug efflux, hyperproduction or } \\
\text { derepression of Amber class C (AmpC), beta-lactamases, } \\
\text { carbapenemase (amber classes A, B, and D) production }\end{array}$ \\
\hline & & \\
\hline & Aminoglycosides & Diminished drug entry, aminoglycoside modifying enzymes \\
\hline & Fluoroquinolones & Point mutations at topoisomerase binding sites (gyr A or par C) \\
\hline
\end{tabular}




\section{Epidemiology of resistant Gram-negative bacterial infections}

Over the last decade, Enterobacteriaceae carrying ESBLbearing plasmids (primarily E. coli and Klebsiella spp.) have become the dominant cause of resistance in Gram-negative bacteria (GN) in both hospital and community settings [25]. Several specific ESBL-carrying plasmids, named CTX-Ms, currently represent the most predominant mechanism of resistance among GNB and are endemic in much of Asia, Europe, and South America [26]. Data from a large retrospective survey (2005 to 2011), primarily among European centers, revealed that ESBLs accounted for $34 \%$ of Gram-negative bacteremic episodes in adult cancer patients, although regional incidence varied widely throughout Europe [2]. A recent study of 350 cases of $E$. coli bacteremia in cancer patients revealed that the overall 30-day mortality rate was higher among patients with ESBL $E$. coli bacteremia than for those with susceptible $E$. coli strains (22.1 vs. $12.2 \% ; p=0.02$ ) [27]. In a study from South Korea, ESBL-associated bacteremia was identified as an independent risk for mortality in patients with hematologic malignancies and associated with a 30-day mortality of almost $45 \%$ [28]. Although many ESBLs produced by $E$. coli are inhibited by $\beta$-lactamase inhibitors such as tazobactam, piperacillin is not consistently active and carbapenems remain the treatment of choice for ESBL-related infections; delay of early adequate antibiotic therapy still correlates with increased mortality outcomes [25, 29-31]. Unfortunately, frequent co-carriage of other antibiotic resistance genes on ESBL-expressing plasmids has led to increasing multidrug resistance including the fluoroquinolones [32].

Carbapenem-resistant Enterobacteriaceae or CREs (primarily $K$. pneumoniae and E. coli) are most commonly isolated and include the $K$. pneumoniae carbapenemases (KPCs). Metallo- $\beta$-lactamases (NDM, VIM, IMP, OXA) are another group of plasmid-mediated carbapenemases that have emerged in K. pneumoniae and E. coli. P. aeruginosa- and Acinetobacter spp.-containing carbapenemases are often multidrug resistant [33]. Alarmingly, CREs and other carbapenemases can hydrolyze all penicillins, cephalosporins, and aztreonam, as well as the carbapenems. Usually, they remain susceptible only to colistimethate and tigecycline. One report from New York City documented a $56 \%$ mortality rate among 18 patients with HM who developed CRE bacteremias [34]. In a systematic literature review evaluating pathogens causing bacteremias in oncology patients, Mikulska et al. [2] found that $20 \%(11-72 \%)$ of Gramnegative isolates were resistant to carbapenems, including $44 \%(3-66 \%)$ of $P$. aeruginosa isolates. Two recent publications from Turkey and Greece describe high rates of 30-day mortality among cancer patients who developed CRE bacteremias $[35,36]$.

\section{Multidrug resistance}

Multiple drug-resistant (MDR) Gram-negative isolates in cancer patients are frequent among the "non-fermenters," $P$. aeruginosa, Acinetobacter spp., and $S$. maltophilia (the last being intrinsically resistant to carbapenems). MDR is now defined as resistance to at least one antibiotic in three different classes. A recent study reported by Cattaneo et al. included 441 episodes of bacteremia in neutropenic patients with HM occurring between 2004 and 2010, 66 were due to $P$. aeruginosa, among which $33 \%$ were MDR [37]. Inadequate empiric treatment was associated with a mortality rate of $83 \%$ in these cases. In a multivariate analysis, history of chronic obstructive pulmonary disease and carbapenem use were identified as risk factors for developing MDR P. aeruginosa infection [38].

In a prospective study of cancer patients at a Spanish referral center, Guidol et al. found that $13.7 \%$ of bacteremia episodes were due to MDR Gram-negative organisms, although their definition included resistance to only two antibiotic classes [39]. In this study, the occurrence of MDR GNB was an independent risk factor for increased 30-day mortality (OR 3.5, $95 \%$ CI 1.4-9.1). Substantial variation in the frequency of MDR isolation by geographic location within Europe was reported [2], with rates among GNB being significantly higher in eastern and southern European countries, including Greece, Italy, Spain, Turkey, Russia, Romania, Hungary as well as Israel. Not surprisingly, MDR pathogens are often found to occur as "breakthrough infections" in patients currently or recently receiving antibiotics [39-41]. In the USA, Rangaraj et al. [41] reported that MDR Gram-negative bacteria, including $P$. aeruginosa and $E$. coli, accounted for approximately $10 \%$ of breakthrough bacteremias among cancer patients who were receiving antibiotics. The observed high all-cause mortality among cancer patients with invasive CRE infections is linked to limited antimicrobial options for treatment. $A$. baumannii infection is uncommon, but resistance to antimicrobials is very high, with MDR strains representing more than $80 \%$ in some cases. The incidence of S. maltophilia is very low but has increased in some institutions, especially in the presence of prolonged neutropenia, mechanical ventilation, and selective pressure by carbapenems [42].

\section{Risk factors for bacteremia caused by antibiotic-resistant bacteria}

Colonization with resistant organisms, immunosuppressed status, and recent exposure to broad-spectrum antibiotics are major risk factors for development of invasive disease. Specifically, in a study by Bodro et al., factors independently associated with resistant ESKAPE bacteremia included medical comorbidities, prior antibiotic therapy, a urinary catheter, and a urinary tract source [5]. In a German prospective study 
among HM and oncology patients, ESBLs or VRE stool colonization was associated with subsequent ESBL bloodstream infection (BSI) [RR 4.5, $95 \%$ CI 2.89-7.04] and VRE BSI (RR 10.2, 95 \% CI 7.87-13.32), respectively [21]. Acute myelogenous leukemia and prior treatment with fluoroquinolones were identified as independent risk factors for ESBL BSI in colonized patients. In other studies, risk factors associated with the development of ESBL-associated bacterial infections also include prior use of cephalosporins or fluoroquinolones, as well as severe illness and recent hospitalization [27, 28, 43]. Risk factors for MDR in neutropenic patients with HM or HSCT include the presence of medical comorbidities, prior antibiotic therapy, and the presence of a urinary catheter [39]. Unfortunately, all these risk factors are quite common and provide little specificity to identify which patients will most likely develop invasive-resistant bacterial infections.

\section{Treatment options: Gram-positive bacterial infections}

Methicillin-resistant S. aureus (MRSA), methicillin-resistant Staphylococcus epidermidis (MRSE), vancomycin-resistant Enterococcus species (VRE), and viridans group streptococci are among the most important pathogens that cause serious infections in at-risk patients. MRSA infections are most frequently treated with vancomycin, and this agent also has been combined with several antibiotics in the search for in vitro synergism or enhanced activity, including oxacillin, nafcillin, cefazolin, ceftaroline, imipenem, rifampin, gentamicin, quinupristin/dalfopristin, and clindamycin. There is some evidence that vancomycin plus linezolid might be antagonistic [44]. Although vancomycin plus rifampin might be attractive because of pharmacodynamic considerations (e.g., vancomycin acting extracellularly and rifampin intracellularly), there is concern that rifampin resistance might arise during therapy and that rifampin might prolong bacteremia and increase both hepatic adverse effects and drug interactions [45].

Daptomycin is finding increasing use in neutropenic patients [46] and has been studied in combination with cloxacillin, nafcillin, oxacillin, ceftaroline, rifampin, gentamicin, clarithromycin, fosfomycin, and trimethoprim-sulfamethoxazole. Yang et al. have described a "seesaw" effect with daptomycin plus oxacillin where the development of daptomycin resistance in MRSA was accompanied by a decrease in oxacillin resistance [47]. A clinical report suggested that antistaphylococcal $\beta$-lactam drugs in combination with daptomycin might enhance clearance of bacteremia due to daptomycin-resistant strains [48]. Another report showed enhanced bacterial clearance of a daptomycin non-susceptible MRSA strain with daptomycin plus ceftaroline in a patient with bacterial endocarditis [49].

Linezolid has been studied in various models in combination with a carbapenem, high-dose daptomycin, rifampin, and doxycycline against MRSA, MRSE, and enterococci [50], but clinical studies are limited. New drugs for use against MRSA infections include telavancin, ceftobiprole, tedizolid, oritavancin, and dalbavancin, some of which are in various stages of development in different countries.

Vancomycin-resistant enterococci may be amenable to treatment with high-dose daptomycin (>6 mg/kg/day), fosfomycin, quinupristin/dalfopristin, linezolid (bacteriostatic), tigecycline (bacteriostatic), and possibly ceftaroline and oritavancin [51].

\section{Treatment options: Gram-negative bacterial infections}

In the face of increasing resistance among GNB in FNP, continued re-evaluation of the traditional empiric approach will be necessary. It is possible that monotherapy might not be adequate for these patients and novel combinations will be needed [52].

Multiple drug-resistant (MDR) Gram-negative bacteria pose the greatest risk to neutropenic and other immunocompromised patients. Treatment of carbapenem-resistant Gramnegative infections is complex and poorly understood. Experience is limited and is based on retrospective studies in non-neutropenic patients. It shows that a combination of two or more active drugs (i.e., colistin, tigecycline, or fosfomycin) with a carbapenem is associated with a better outcome [53].

Definitive controlled studies in neutropenic patients are lacking, but CREs have been studied in vitro with antibiotic combinations including colistin or polymyxin B with an aminoglycoside, tigecycline, doxycycline, rifampin, fosfomycin, and daptomycin. A recent retrospective study from three Italian centers that included 126 patients with $\mathrm{KpC}$ bacteremia (non-neutropenic) showed lower mortality with the triple combination of colistin plus tigecycline plus meropenem than with single-agent treatment (34 vs. $54 \%, p=0.02$ ) [54]. Fosfomycin may show in vitro synergism against CRE with carbapenems, colistin, aminoglycosides, and tigecycline [55].

An intriguing suggestion from in vitro and experimental models implies that a double carbapenem combination might hold promise for the treatment of MDR Gram-negatives including CRE. Bulik and Nikolau [56] combined ertapenem (a carbapenem with little or no activity against these organisms, but for which these organisms' carbapenemase has a high affinity) with the more active doripenem. Ertapenem acted as a "decoy" target for the carbapenemase, leaving doripenem relatively more able to act against the organisms. A preliminary report from this group at the 2013 ECCMID meeting suggested that this combination produced better clinical results against $\mathrm{KpC}$ bacteremia than the combination of colistin plus doripenem [57].

With the increasing dissemination of MDR and XDR Gramnegative bacteria around the world, the development of new agents potentially active against these organisms is becoming critically important. Boucher et al. [58] have summarized the 
current candidate drugs under development including ceftaroline/tazobactam, ceftozolane/tazobactam, ceftaroline/ avibactam, ceftazidime/avibactam, imipenem/MK-7655 (a new beta-lactamase inhibitor), plazomycin, eravacycline, and brilacidin. Some of these drugs have already been introduced but there are little or no published data about use in FNP.

\section{Novel approaches are needed}

Until clinical trials of newly introduced antibiotics are established in febrile neutropenic patients, it is important to optimize the use of currently available antimicrobial agents. Optimizing pharmacodynamics (effect of drugs on the organisms) has been studied in in vitro pharmacokinetic models with the goal of identifying the clinical dose that will provide maximal antimicrobial effect and minimal selection of bacterial resistance [59].

In some difficult clinical situations, strategies such as prolonged or continuous infusions of antibiotics and the use of aerosolized antibiotics applied directly to the respiratory tract deserve consideration, although results from the very few prospective trials are inconsistent.

Beyond these considerations, there are a series of new developments that could provide some hedge against the increasing threat of resistant organisms that might not be solved with the use of combinations of presently available antibiotics or by pharmacological manipulation of these drugs. These include drugs that can attenuate the virulence of bacteria in vivo, chemoprotectants, retinoid receptor agents, and chemokine receptor-4 agonists. Better understanding of natural immunity and its possible enhancement could represent another approach. As an example, antimicrobial proteins and antiinfective peptides from mammalian leukocytes have been developed as potential therapeutic agents and have entered clinical trials.

\section{Conclusions}

We are facing a major challenge as a result of the continued emergence of multiresistant microorganisms, and we risk returning to the pre-antibiotic era with few or limited active agents. As new agents with activity against resistant Grampositive and Gram-negative bacteria are introduced, they should be carefully studied in febrile neutropenic infections. As these developments are awaited, we urge consideration of local antibiotic susceptibility data in selecting empiric combination therapy that will provide the most active agents for the resistant pathogens discussed in this review. Pressure on the pharmaceutical industry to discover and evaluate novel agents that might be active against multidrug-resistant pathogens should continue in an attempt to mitigate the adverse outcomes in these fragile patients.

\section{Compliance with ethical standards}

Conflict of interest The authors declare that they have no conflicts of interest.

\section{References}

1. Montassier E, Batard E, Gastinne T, et al. (2013) Recent changes in bacteremia in patients with cancer: a systematic review of epidemiology and antibiotic resistance. Eur J Clin Microbiol Infect Dis 32(7):841-850

2. Mikulska M, Viscoli C, Orasch C, et al. (2014) Aetiology and resistance in bacteraemias among adult and paediatric haematology and cancer patients. J Infect 68(4):321-331

3. Rice LB (2008) Federal funding for the study of antimicrobial resistance in nosocomial pathogens: No ESKAPE. J Infect Dis 197(8):1079-1081

4. Bodro M, Sabé N, Tubau F, et al. (2013) Risk factors and outcomes of bacteremia caused by drug-resistant ESKAPE pathogens in solid-organ transplant recipients. Transplantation 96(9):843-849

5. Bodro M, Gudiol C, Garcia-Vidal C, et al. (2014) Epidemiology, antibiotic therapy and outcomes of bacteremia caused by drugresistant ESKAPE pathogens in cancer patients. Support Care Cancer 22(3):603-610

6. Rolston KV, Yadegarynia D, Kontoyiannis DP, et al. (2006) The spectrum of Gram-positive bloodstream infections in patients with hematologic malignancies, and the in vitro activity of various quinolones against Gram-positive bacteria isolated from cancer patients. Int J Infect Dis 10(3):223-230

7. Baos E, Candel FJ, Merino P, et al. (2013) Characterization and monitoring of linezolid-resistant clinical isolates of Staphylococcus epidermidis in an intensive care unit 4 years after an outbreak of infection by cfr-mediated linezolid-resistant Staphylococcus aureus. Diagn Microbiol Infect Dis 76(3): 325-329

8. Bayer AS, Schneider T, Sahl HG (2013) Mechanisms of daptomycin resistance in Staphylococcus aureus: role of the cell membrane and cell wall. Ann N Y Acad Sci 1277:139-158

9. Rice LB (2006) Antimicrobial resistance in gram-positive bacteria. Am J Infect Control 34(5 Suppl 1):S11-S19 discussion $\mathrm{S} 64-73$

10. Wisplinghoff H, Reinert RR, Cornely O, et al. (1999) Molecular relationships and antimicrobial susceptibilities of viridans group streptococci isolated from blood of neutropenic cancer patients. J Clin Microbiol 37(6):1876-1880

11. Sader HS, Jones RN, Rossi KL, et al. (2009) Occurrence of vancomycin-tolerant and heterogeneous vancomycin-intermediate strains (hVISA) among Staphylococcus aureus causing bloodstream infections in nine USA hospitals. J Antimicrob Chemother 64(5):1024-1028

12. Nesher L, Rolston KV (2014) The current spectrum of infection in cancer patients with chemotherapy related neutropenia. Infection 42(1):5-13

13. Gudiol C, Bodro M, Simonetti A, et al. (2013) Changing aetiology, clinical features, antimicrobial resistance, and outcomes of bloodstream infection in neutropenic cancer patients. Clin Microbiol Infect 19(5):474-479

14. Mihu CN, Rhomberg PR, Jones RN, et al. (2010) Escherichia coli resistance to quinolones at a comprehensive cancer center. Diagn Microbiol Infect Dis 67(3):266-269 
15. Pitout JD, Laupland KB (2008) Extended-spectrum beta-lactamase producing Enterobacteriaceae: an emerging public-health concern. Lancet Infect Dis 8(3):159-166

16. Patel G, Bonomo RA (2013) "Stormy waters ahead": global emergence of carbapenemases. Front Microbiol 4:48

17. Malhotra-Kumar S, Xavier BB, Das AJ et al. (2016) Colistin resistance gene $\mathrm{mcr}-1$ harboured on a multidrug resistant plasmid. Lancet Infect Dis. Epub Jan 7, 2016

18. Tenover F, Goering RV (2009) Methicillin-resistant Staphylococcus aureus strain USA 300: origin and epidemiology. J Antimicrob Chemother 64:441-446

19. Figuera Esparza M, Carballo M, et al. (2006) Microbiological isolates in patients with febrile neutropenia and hematological neoplasias. Revista Espanola de Quimioterapia 19(3):247-251

20. Mahajan SN, Shah JN, Hachem R, et al. (2012) Characteristics and outcomes of methicillin-resistant Staphylococcus aureus bloodstream infections in patients with cancer treated with vancomycin: 9 -year experience at a comprehensive cancer center. Oncologist 17(10):1329-1336

21. Liss BJ, Vehreschild JJ, Cornely OA, et al. (2012) Intestinal colonisation and blood stream infections due to vancomycin-resistant enterococci (VRE) and extended-spectrum beta-lactamaseproducing Enterobacteriaceae (ESBLE) in patients with haematological and oncological malignancies. Infection 40(6):613-619

22. Doern GV, Ferraro MJ, Brueggemann AB, et al. (1996) Emergence of high rates of antimicrobial resistance among viridans group streptococci in the United States. Antimicrob Agents Chemother 40(4):891-894

23. Marron A, Carratala J, Fet A, et al. (2001) High rates of resistance to cephalosporins among viridans-group streptococci causing bacteraemia in neutropenic cancer patients. J Antimicrob Chemother 47(1):87-91

24. Bruckner L, Gigliotti F (2006) Viridans group streptococcal infections among children with cancer and the importance of emerging antibiotic resistance. Semin Pediatr Infect Dis 17(3):153-160

25. Savard P, Perl T (2012) A call to action: managing the emergence of multidrug-resistant Enterobacteriaceae in the acute care settings. Curr Opin Infect Dis 25:371-377

26. Woodford N, Carattoli A, Karisik E, et al. (2009) Complete nucleotide sequences of plasmids pEK204, pEK499, and pEK516, encoding CTX-M enzymes in three major Escherichia coli lineages from the United Kingdom, all belonging to the international O25: H4-ST131 clone. Antimicrob Agents Chemother 53(10):44724482

27. Ha YE, Kang C, Cha MK, et al. (2013) Epidemiology and clinical outcomes of bloodstream infections caused by extended-spectrum $\beta$-lactamase-producing Escherichia coli in patients with cancer. Int J Antimicrob Agents 42(5):403-409

28. Kang CI, Chung DR, Ko KS, et al. (2012) Korean Network for Study of Infectious Diseases. Risk factors for infection and treatment outcome of extended-spectrum beta-lactamase-producing Escherichia coli and Klebsiella pneumoniae bacteremia in patients with hematologic malignancy. Ann Hematol 91(1):115-121

29. Gudiol C, Calatayud L, Garcia-Vidal C, et al. (2010) Bacteraemia due to extended-spectrum beta-lactamase-producing Escherichia coli (ESBL-EC) in cancer patients: clinical features, risk factors, molecular epidemiology and outcome. J Antimicrob Chemother 65(2):333-341

30. Kim SH, Kwon JC, Choi SM, et al. (2013) Escherichia coli and Klebsiella pneumoniae bacteremia in patients with neutropenic fever: factors associated with extended-spectrum beta-lactamase production and its impact on outcome. Ann Hematol 92(4):533-541

31. Tumbarello M, Spanu T, Sanguinetti M, et al. (2006) Bloodstream infections caused by extended-spectrum-beta-lactamase-producing klebsiella pneumoniae: risk factors, molecular epidemiology, and clinical outcome. Antimicrob Agents Chemother 50(2):498-504
32. Trecarichi EM, Tumbarello M, Spanu T, et al. (2009) Incidence and clinical impact of extended-spectrum-beta-lactamase (ESBL) production and fluoroquinolone resistance in bloodstream infections caused by Escherichia coli in patients with hematological malignancies. J Infect 58(4):299-307

33. Hawkey PM, Jones AM (2009) The changing epidemiology of resistance. J Antimicrob Chemother 64(Suppl 1):i3-10

34. Satlin MJ, Calfee DP, Chen L, et al. (2013) Emergence of carbapenem-resistant Enterobacteriaceae as causes of bloodstream infections in patients with hematologic malignancies. Leuk Lymphoma 54(4):799-806

35. Dizbay M, Guzel Tunccan O, et al. (2014) Emergence of carbapenem-resistant Klebsiella spp. infections in a Turkish university hospital: epidemiology and risk factors. J Infect Dev Ctries 8(1):44-4915

36. Falagas ME, Tansarli GS, Karageorgopoulos DE, et al. (2014) Deaths attributable to carbapenem-resistant Enterobacteriaceae infections. Emerg Infect Dis 20(7):1170-1175

37. Cattaneo C, Antoniazzi F, Casari S, et al. (2012) P. aeruginosa bloodstream infections among hematological patients: an old or new question? Ann Hematol 91(8):1299-1304

38. Ohmagari N, Hanna H, Graviss L, et al. (2005) Risk factors for infections with multidrug-resistant Pseudomonas aeruginosa in patients with cancer. Cancer 104(1):205-212

39. Gudiol C, Tubau F, Calatayud L, et al. (2011) Bacteraemia due to multidrug-resistant gram-negative bacilli in cancer patients: risk factors, antibiotic therapy and outcomes. J Antimicrob Chemother 66(3):657-663

40. Oliveira AL, de Souza M, Carvalho-Dias VM, et al. (2007) Epidemiology of bacteremia and factors associated with multidrug-resistant gram-negative bacteremia in hematopoietic stem cell transplant recipients. Bone Marrow Transplant 39(12):775-781

41. Rangaraj G, Granwehr BP, Jiang Y, et al. (2010) Perils of quinolone exposure in cancer patients: breakthrough bacteremia with multidrug-resistant organisms. Cancer 116(4):967-973

42. Safdar A, Rolston KV (2007) Stenotrophomonas maltophilia: changing spectrum of a serious bacterial pathogen in patients with cancer. Clin Infect Dis 45(12):1602-1609

43. Ortega M, Marco F, Soriano A, et al. (2009) Analysis of 4758 Escherichia coli bacteraemia episodes: predictive factors for isolation of an antibiotic-resistant strain and their impact on the outcome. J Antimicrob Chemother 63(3):568-574

44. Singh SR, Bacon AE, Young DC, et al. (2009) In vitro 24-hour time-kill studies of vancomycin and linezolid in combination versus methicillin-resistant Staphylococcus aureus. Antimicrob Agents Chemother 54:4495-4497

45. Tremblay S, Lau TTY, Ensom MHH (2013) Addition of rifampin to vancomycin for methicillin-resistant Staphylococcus aureus infections: what is the evidence? Ann Pharmacother 47:1045-1064

46. Rolston KV, McConnell SA, Brown J, et al. (2010) Daptomycin use in patients with cancer and neutropenia: data from a retrospective registry. Clin Adv Hematol Oncol 8:249-256

47. Yang S-J, Xiong YO, Boyle-Vavra S, et al. (2010) Daptomycinoxacillin combinations in treatment of experimental endocarditis caused by daptomycin-nonsusceptible strains of methicillin-resistant Staphylococcus aureus with evolving oxacillin susceptibility (the "seesaw effect"). Antimicrob Agents Chemother 54:3161-3169

48. Dhand A, Bayer AS, Pogliano J, et al. (2011) Use of antistaphylococcal $\beta$-lactams to increase daptomycin activity in eradicating persistent bacteremia due to methicillin-resistant Staphylococcus aureus: role of enhanced daptomycin binding. Clin Infect Dis 53:158-163

49. Rose WE, Schulz LT, Andes D, et al. (2012) Addition of ceftaroline to daptomycin after emergence of daptomycin-nonsusceptible Staphylococcus aureus during therapy improves antibacterial activity. Antimicrob Agents Chemother 56:5296-5302 
50. Smirnova MV, Strukova EN, Portnoy YA, et al. (2011) The antistaphylococcal pharmacodynamics of linezolid alone and in combination with doxycycline in an in vitro dynamic model. $\mathrm{J}$ Chemother 23:140-144

51. Rivera AM, Boucher HW (2011) Current concepts in antimicrobial therapy against select gram-positive organisms: methicillinresistant Staphylococcus aureus, penicillin-resistant pneumococci and vancomycin-resistant enterococci. Mayo Clinic Proc 86: $1230-1243$

52. Klastersky J, Georgala A (2014) Strategies for the empirical management of infection in cancer patients with emphasis on the emergence of resistant Gram-negative bacteria. Crit Rev Oncol Hematol 92(3):268-278

53. Akova M, Daikos GL, Tzouvelekis L, et al. (2012) Interventional strategies and current clinical experience with carbapenemaseproducing gram-negative bacteria. Clin Microbiol Infect 18(5): $439-448$

54. Tumbarello M, Viale P, Viscoli C, et al. (2012) Predictors of mortality in bloodstream infections caused by Klebsiella pneumoniae carbapenemase-producing K. pneumoniae: importance of combination therapy. Clin Infect Dis 55:943

55. Samonis G, Maraki S, Karageorgopoulos DE, et al. (2012) Eur J Jlin Micro Infect Dis 31:695-701

56. Bulik CC, Nicolau DP (2011) Double-carbapenem therapy for carbapenemase-producing Klebsiella pneumoniae. Antimicrob Agents Chemother 55:3002-3004

57. Nogid B, Venugopolan V, Bias T, et al. (2013) Double carbapenem therapy for bacteremia due to carbapenem resistant Klebsiella pneumonia: from test tube to clinical practice. Poster Presentation ECCMID, Berlin, Germany

58. Boucher HW, Talbot GH, Benjamin DK Jr, et al. (2013) $10 \times$ '20 Progress - development of new drugs active against gram-negative bacilli: an update from the Infectious Diseases Society of America. Clin Infect Dis 56:1685-1694

59. Firsov AA, Portnoy YA, Strukova EN, et al. (2014) Predicting bacterial resistance using the time inside the mutant selection window: possibilities and limitations. Int $\mathbf{J}$ Antimicrob Agents 44:301-305 